\title{
Theory of superconductor-insulator transition in single Josephson junctions
}

\author{
S.G. Chung \\ Max-Planck-Institut für Physik komplexer Systeme, Nöthnizer Str. 38, D-01187 Dresden, Germany
}

\begin{abstract}
A non-band theory is developed to describe the superconductor-insulator (SI) transtition in resistively shunted, single Josephson junctions. The $I-V$ characteristic is formulated by a Landauerlike formula and evaluated by the path-integral transfer-matrix method. The result is consistent with the recent experiments at around $80 \mathrm{mK}$. However, the insulator phase shrinks with decreasing temperature indicating that the single Josephson junction becomes all superconducting at absolute zero temperature, as long as dissipation is present.
\end{abstract}

74.50.+r,73.23.Hk,73.40.Gk

The resistively shunted single Josephson junction(RSJ) at $T=0$ is described classically by the second order differential equation for the phase difference between the two superconductors,

$$
C \frac{\hbar \ddot{\phi}}{2 e}+\frac{1}{R_{\mathrm{T}}} \frac{\hbar \dot{\phi}}{2 e}+I_{0} \sin \phi=I
$$

Here $C$ is the capacitance, $R_{T}$ is the normal state resistance of the junction, $I_{0}$ is the critical current, and $I$ is the bias current. A mechanical analogy of a particle moving in a tilted washboard potential with friction may be helpful. The $I-V$ characteristic resulting from (1) was studied by Stewart 11. The capacitance brings about hysteresis. In the weak dissipation limit $R_{\mathrm{T}} \rightarrow \infty$ current is entirely carried by Cooper pairs at $V=0$ and up to the critical current. This is the dc Josephson effect. For $I>I_{0}$, the $I-V$ curve switches to the Ohmic branch where current is entirely carried by electrons, $V=R I$. In the opposit limit $R_{\mathrm{T}} \rightarrow 0$ hysteresis disappears and supercurrent at $V=0$ is gradualy replaced by the normal current with increasing voltage. The thermal and quantum fluctuation is expected to affect only the regime $I / I_{0} \ll R_{q} / R_{T} \equiv g$, where $R_{q} \equiv h / 4 e^{2}=6.45 K \Omega$ is the quantum resistance. Now for a typical charging energy of $1 \mathrm{~K}$, the critical current is $I_{0}=2 e E_{J} / \hbar=42 E_{J} / E_{C} n A$ whereas the parameter ranges associated with the SI transition are $g \lesssim 1, E_{J} / E_{C} \lesssim 1$. The range of current where fluctuation is important is thus up to a few $n A$. The focus of the present paper is precisely in this current regime. It should be noted that if $g \gg 1$, the bias current could be as large as the critical current while still staying in the regime where fluctuation is important. Indeed this case was studied theoretically [2] 3] and experimentally [4] focusing on the negative differential resistance as a signal of the Coulomb blockade of Cooper pair tunneling.

A microscopic description is to take into account the quasi-particle tunneling but with zero superconducting gap (resistively shunted) as a source of dissipation. The path-integral expression of the partition function is [5],

$$
Z_{0}=\int \mathcal{D} \phi \exp (-S / \hbar)
$$

$$
\begin{aligned}
S= & \int_{0}^{\beta \hbar} d \tau\left[\frac{C}{2}\left(\frac{\hbar}{2 e} \dot{\phi}\right)^{2}+U(\phi)\right] \\
& -\int_{0}^{\beta \hbar} \int_{0}^{\beta \hbar} d \tau d \tau^{\prime} \alpha\left(\tau-\tau^{\prime}\right) \cos \left(\frac{\phi(\tau)-\phi\left(\tau^{\prime}\right)}{2}\right)
\end{aligned}
$$

where

$$
\begin{array}{r}
U(\phi)=-E_{J} \cos \phi-\frac{I \hbar}{2 e} \phi \\
\alpha(\tau)=\frac{\hbar}{2 \pi e^{2} R_{\mathrm{T}}} \frac{(\pi / \beta \hbar)^{2}}{\sin ^{2}(\pi \tau / \beta \hbar)}
\end{array}
$$

The single Josephson junction (JJ) has also been studied by a phenomenological model [6] which is just to replace the term $\cos \left(\frac{\phi(\tau)-\phi\left(\tau^{\prime}\right)}{2}\right)$ in (3) by $\phi(\tau) \phi\left(\tau^{\prime}\right)$ leading, upon stationally phase approximation, to (1) with retarded dissipation.

The first question concerning the phase dynamics is whether it is coherent or incoherent. A band theory assumes a coherent motion of phase over many local minima of the washboard potential. In band picture, the voltage calculation is easy because it is proportional to the group velocity of the quasi-charge [2]. On the other hand, a non-band theory is appropriate for incoherent motion, and the problem is reduced to the calculation of the escape rate of particle out of a dissipative metastable well, the quantum Kramers rate.

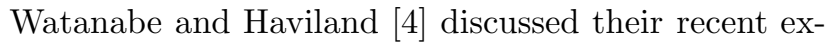
periment on the Coulomb blockade at $g \gg 1$ in light of the band theory. However, other experiments [7,8] have not found the negative differential resistance in the SI transition regime $g \lesssim 1$. This indicates that the phase dynamics near the SI transition is dominated by incoherent tunneling. Indeed a typical experimental setup is, $E_{C}=1 \mathrm{~K}, E_{J} / E_{C}=0.1 \sim 20, T / E_{C}=0.1 \sim 1$ and $g \lesssim 1$. And most importantly, the minimum voltage experimentally observable is of order $0.5 \mu \mathrm{V}$ [7] which translates to the temperature $10 \mathrm{mK}$. So when the energy splitting due to tunneling exceeds this value, the corresponding state is experimentally recognized as insulating. However, $10 \mathrm{mK}$ is much smaller than the potential barrier $E_{J}$ at the SI transition. Near the SI transition 
at $g=0$, therefore, the effect of many wells is expected unimportant. Switching on dissipation, the SI transition shifts to a smaller critical $E_{J} / E_{C}$, but then dissipation will suppress the effect of incresed tunneling strength. One thus expects that the phase dynamics essentially at a single well would determine the SI transition.

In this paper we propose a non-band theory for the SI transition [9]. Two methods have been employed to calculate the quantum Kramers rate along this line. One is the imaginary free energy method with an instanton technique [6,10]. The other is the quntum transition state (QTS) theory long known in chemistry [11,12]. Here we develop a new approach combining a Landauer-like formula for the quantum Kramers rate 13] and the cluster transfer matrix (TM) method 114. The Landauer formula for electrical conductance has proven to be quite useful in mesoscopic systems. In the quantum Kramers rate problem, there is formally exact formula [15] analogous to the Green-Kubo-Nakano formula for electrical conductivity. The corresponding Landauer-like formula is the quantum transient state (QTS) theory which tries to calculate the Kramers rate by summing up all the probabilities of finding particle at the top of the barrier which have positive, outgoing momentum. The QTS theory, however, has often been exercised with a semiclassical approximation 12,16 and its drawbacks do not show up this way. As soon as one tries to exactly evaluate the path-integrals, however, one encounters difficultiess. First, the contribution to the escape rate is no longer local. The phase motion at different places than at the barrier top contributes. Moreover, the non-local contribution could become negative, as discussed in [13]. This undesirable nonlocality and negativity originates from the Weyle quantization procedure 10,11]. The original idea to collect all the outgoing, positive contribution at the barrier site, is thus spoiled.

We have recently proposed a Landauer-like formula for the quantum Kramers rate. Applied to the voltage calculation here, it reads 13.

$$
\frac{V}{e / 2 C}=[1-\exp (-\pi I \hbar \beta / e)] \times \frac{1}{2} \sqrt{\left\langle\delta\left(\phi-\phi_{0}\right) P^{2} / L\right\rangle}
$$

where $P \equiv-\partial_{\phi}, \phi_{0}$ is the barrier top in the potential $U(\phi), L$ is the system size, and $\langle\ldots\rangle$ is a thermal average. The first factor in (5) takes into account the backward flux by detailed balance, whereas the second term is esentially square root of average of the kinetic energy, in mechanical analogy, at the barrier top with factor $1 / 2$ taking into account only the right-going contribution. In contrast, the QTS formula is obtained from (5) by replacing $\sqrt{\left\langle\delta\left(\phi-\phi_{0}\right) P^{2} / L\right\rangle} \rightarrow\left\langle\delta\left(\phi-\phi_{0}\right)|P|\right\rangle$. The difference between the formula (5) and the QTS theory is thus a fluctuation which is precisely the undesirable non-local, possibly negative contributions in the QTS theory described in the above. After some manipulations, one can express the average in (5) as

$$
\begin{array}{r}
\left\langle\delta\left(\phi-\phi_{0}\right) P^{2}\right\rangle=Z / Z_{0} \\
Z=-\left.\frac{1}{4} \partial_{\phi}^{2} W\left(\phi, 2 \phi_{0}-\phi\right)\right|_{\phi}=\phi_{0} \\
Z_{0}=\int d \phi W(\phi, \phi)
\end{array}
$$

in terms of the path integral

$$
W\left(\phi, \phi^{\prime}\right)=\int_{\phi \rightarrow \phi^{\prime}} \mathcal{D} \tilde{\phi} \exp (-S / \hbar)
$$

where $\tilde{\phi}(0)=\phi$ and $\tilde{\phi}(\beta \hbar)=\phi^{\prime}$.

The path-integral (9) with the action $S$ given by (3) can be evaluated precisely by the cluster TM method [14. In the present problem, however, the dimensionless conductance is at $g \lesssim 1$, and from the study of the single electron box which has a similar action as (3), this regime of $g$ can be accurately handled by the 1-cluster TM method as follows. First divide the interval $(0, \beta \hbar)$ into $N$ segments of size $\Delta=\beta \hbar / N$. Denoting the discrete phase points as $\phi_{i}, i=1,2, \ldots, N+1$, we have

$$
\exp (-S / \hbar) \equiv \prod_{i=1}^{N} K\left(\phi_{i}, \phi_{i+1}\right) \times \mathrm{Rm}
$$

where the TM operator $K$ is defined by

$$
\begin{aligned}
K= & \exp \left[-\frac{1}{16 \Delta}\left(\phi_{i}-\phi_{i+1}\right)^{2}+J \Delta\left(\cos \phi_{i}+I \phi_{i}\right)\right. \\
& \left.-2 g \frac{\Delta^{2} T^{2}}{\sin ^{2}(\pi T \Delta)}\left(1-\cos \frac{\phi_{i}-\phi_{i+1}}{2}\right)\right]
\end{aligned}
$$

with dimensionless parameters $J \equiv E_{J} / E_{C}, T \equiv$ $k_{B} T / E_{C}, I \equiv I \hbar /(2 e J)$ and

$$
\mathrm{Rm} \equiv \exp \left[-2 g T^{2} \Delta^{2} \sum_{j-i \geq 2}^{N} \frac{1-\left\langle\cos \frac{\phi_{j}-\phi_{i}}{2}\right\rangle}{\sin ^{2}(\pi T \Delta(j-i))}\right]
$$

where the cumulant approximation was used which is precise for $g \lesssim 1$. Second write

$$
\int_{\phi \rightarrow \phi^{\prime}} \mathcal{D} \tilde{\phi}=\prod_{i=1}^{N+1} \int d \phi_{i} \delta\left(\phi_{1}-\phi\right) \delta\left(\phi_{N+1}-\phi^{\prime}\right)
$$

The first $\delta$-function can be written in terms of a complete set $\left\{\psi_{p}\right\}$ as

$$
\delta\left(\phi_{1}-\phi\right)=\sum_{p} \psi_{p}^{*}(\phi) \psi_{p}\left(\phi_{1}\right)
$$

Now one can choose the complete set such that it satisfies the TM equation,

$$
\int_{\phi_{a}}^{\phi_{b}} d \phi K\left(\phi, \phi^{\prime}\right) \psi_{p}(\phi)=\lambda_{p} \psi_{p}\left(\phi^{\prime}\right)
$$


Repeatedly using the TM equation, we arrive at

$$
W\left(\phi, \phi^{\prime}\right)=\mathrm{Rm} \cdot \sum_{p} \lambda_{p}^{N} \psi_{p}^{*}(\phi) \psi_{p}\left(\phi^{\prime}\right)
$$

The correlation function $\left\langle\cos \frac{\phi_{j}-\phi_{i}}{2}\right\rangle$ in (12) can be calculated likewise.

There are three parameters $T, g$ and $E_{J} / E_{C}$. In addition the present method contains two more parameters $\Delta$ and $J_{R}$. The latter defines the phase space; $\phi_{a}=-\pi-\phi_{0}-\pi / J_{R}, \phi_{b}=\pi-\phi_{0}+\pi / J_{R}$. For numerical results obtained, we have checked that the results are affected a couple of $\%$ for the two different $J_{R}=2,3$ and for the two different $\Delta=0.05,0.1$. The results shown below are for $J_{R}=3$ and $\Delta=0.1$. The "zero-bias" resistance reported in experiments may need to be treated carefully. In fact, the voltage measurment in a single JJ is limited, unlike 1D or 2D JJ arrays, to $V_{\min } \sim 0.5 \mu \mathrm{V}[7$. So we have calculated the differential resistance $R \equiv \frac{d V}{d I}$ with the constraint that the voltage is equal to $0.5 \mu \mathrm{V}$ (The calculation is repeated for $V_{\min }=0.05 \mu \mathrm{V}$ to find no essential difference from the results below). At the same time, to be consistent with our theoretical consideration above, we have checked that the current is much smaller than the critical one, $I \ll I_{0}$. For results below, the current $I$ is up to a few $\%$ of the critical one $I_{0}$. Fig. 1 shows a temperature dependence of the resistance at $V=0.5 \mu V$ for $g=0.4$. The small $E_{J} / E_{C}$ case shows an insulator-like temperature dependence $\frac{d R}{d T}<0$, while the large $E_{J} / E_{C}$ case shows a superconductor-like behavior $\frac{d R}{d T}>0$. Repeating the calculations for different $\mathrm{g}$, one can draw a phase diagram. Fig. 2 is a phase diagram at $T=80 \mathrm{mK}$. The experimental results [7, [8] are open circles (superconductor-like) and solid circles (insulator-like) at $T \approx 80 \mathrm{mK}$. Our result is denoted by open diamonds. The band theory result, thick solid line, is obtained by simply replacing the minimum measurament energy $e V_{\min }$ by the thermal energy $k_{B} T$ [7]. The QTS theory result, triangles, is also plotted for comparison. A major disagreement between theory and experiment is for some data points near $g=2.8[8]$. However a similar phase diagram experimentally found for the $2 \mathrm{D}$ JJ arrays with similar parameter ranges for $g, E_{j} / E_{C}$ and $T$ is bounded, $E_{J} / E_{C} \lesssim 0.5$, and $g \lesssim 0.5$ (cf. Fig 3 in $[17]$ ). The above data near $g=2.8$ is currently mysterious.

There are two notable points in the obtained temperature dependence of resistance. First it becomes flat for large $E_{J} / E_{C}$ irrespective of $g$. This is consistent with experimental findings [7]:8]. Theoretically, when the ratio $E_{J} / E_{C}$ is sufficiently large, the quantum Kramers rate would become less dependent on $T$ and $g$. Note a sharp contrast between the single JJ and the 2D JJ arrays [17. The temperature dependence of resistance in the latter case showed a true phase transition behavior, namely resistance decreases or increases roughly exponentially with temperature. Interestingly, the tempera- ture dependence of resistance in 1D JJ arrays also becomes flat deep inside the superconducting phase 18 . The second notable point is that the SI phase boundary defined by vanishing $d R / d T$ actually depends on temperature. In Fig. 3, we have plotted the SI phase boundary for $T=80,60,40,20,10$ and $5 \mathrm{mK}$. Clearly, the insulator phase diminishes with decreasing temperature. We thus reach a conclusion that, as long as dissipation is present, the single JJ becomes all superconducting at absolute zero temperature. Note that, in contrast, the band theory predicts an essentially temperature independent phase boundary. It is interesting to see if our finding can be observed experimentally.

Finally, related to the last point, we should argue that our finding is indeed consistent with the previous theoretical works on a different RSJ model [6,12] and the subsequent experimental observations 19 of a universal $T^{2}$-law for the escape rate $k^{+}$of the zero-voltage state in the single JJ. That is,

$$
\ln \left[k^{+}(T) / k^{+}(0)\right] \propto T^{2} \text { for } T \rightarrow 0
$$

These experiments focused on the highly dissipative regime $g \gg 1$, and the current is very close to the critical current, and therefore the escape rate $k^{+}$is not quite proportional to voltage. In contrast, we focused on the regime $g \lesssim 1$ and calculated voltage since current is only up to a few $\%$ of the critical one. Nevertheless the present theory and the previous theories agree in that both $k^{+}$ and $R(T)$ increase with temperature at very low T. In fact it was argued that the universal low temperature enhancement arizes from the temperature dependence of the heat-bath [12]. In the present case, it is due to the $\alpha(\tau)$ term in the action (3) and its temperature dependence originates from the Fermi distribution function in the electron Green's function [5]. As $T \rightarrow 0$, this term dominates the temperature dependence and the voltage behaves as

$$
\ln [V(T) / V(0)] \propto g T^{2}
$$

leading to $\frac{d R}{d T}>0$ for $T \rightarrow 0$. Note that, as is clear from (18) and seen in Fig. 3, such a temperature dependence disappears at $g=0$.

This work was supported by the Visitor Program of the MPI-PKS. The work was also partially supported by the NSF under Grant No. DMR990002N and utilized the SGI/CRAY Origin2000 at the National Center for Supercomputing Applications at the University of Illinois at Urbana-Champaign. I thank Mikhail Fistul, Pertti Hakonen and Pavel Lipavský for helpful discussions and Chiidon Chen and Watson Kuo for correspondence on their experiment. I also thank Peter Hänggi, Ivan Larkin and Anthony Leggett for enlightening conversations. 
[1] W.C. Stewart, Appl. Phys. Lett. 12, 277(1968).

[2] K.K. Likharev and A.B. Zorin, J. Low Temp. Phys. 59, 347(1985).

[3] G.-L. Ingold and H. Grabert, Phys. Rev. Lett. 83, 3721(1999); H. Gravert and G.-L. Ingold, Superlattices and Microstructures 25, 915(1999).

[4] M. Watanabe and D. Haviland, cond-mat/0103502.

[5] U. Eckern, G. Schön, and V. Ambegaokar, Phys. Rev. B 30, 6419(1984).

[6] H. Grabert and U. Weiss, Phys. Rev. Lett. 53, 1787(1984); U. Weiss, H. Gravert, P. Hänggi and P. Riseborough, Phys. Rev. B 35, 9535(1987).

[7] J.S. Penttilä, Ü. Parts, P.J. Hakonen, M.A. Paalanen and E.B. Sonin, Phys. Rev. Lett. 82, 1004(1999).

[8] R. Yagi, S. Kobayashi and Y. Otuka, J. Phys. Soc. Japan 66, 3722(1997).

[9] A part of the work was reported at the 14th international conference on the electronic properties of 2-D systems, Praha, and a short proceeding will appear in Physica E.

[10] U. Weiss, Quantum Dissipative Systems, 2-nd ed., Series in Modern Condensed Matter Physics, Vol. 10 (World Scientific, Singapore, 1998).

[11] W.H. Miller, J. Chem. Phys. 61, 1823(1974); 62, 1899(1975).

[12] P. Hänggi, P. Talkner and M. Borkovec, Rev. Mod. Phys. 62, 251(1990).

[13] S.G. Chung, unpublished.

[14] S.G. Chung, J. Korea Phys. Soc. 33, S25(1998); J. Phys. Soc. Japan 68, 1778(1999).

[15] T. Yamamoto, J. Chem. Phys., 33, 281(1960); W.H. Miller, S.D. Schwartz and J.W. Tromp, ibid. 79, 4889(1983).

[16] V.I. Mel'nikov, Phys. Rep. 209, 1(1991)

[17] T. Yamaguchi, R. Yagi, A. Kanda, Y. Ootuka and S. Kobayashi, Phys. Rev. Lett. 85, 1974(2000).

[18] D.B. Haviland, K. Anderson and P. Argen, condmat/0001143; W. Kuo, J.-H. Shyu, Y.-J. Chien, D.-S. Chung, M.-L. Jeng and C. Chen, cond-mat/0007355; W. Kuo and C. Chen, cond-mat/0103245.

[19] S.Washburn, R.A. Webb, R.F. Voss and S.M. Faris, Phys. Rev. Lett. 54, 2712(1985); D.B. Schwartz, B. Sen, C.N. Archie and J.E. Lukens, ibid. 55, 1547(1985); A.N. Cleland, J.M. Martinis and J. Clarke, ibid. 37, 5950(1988).

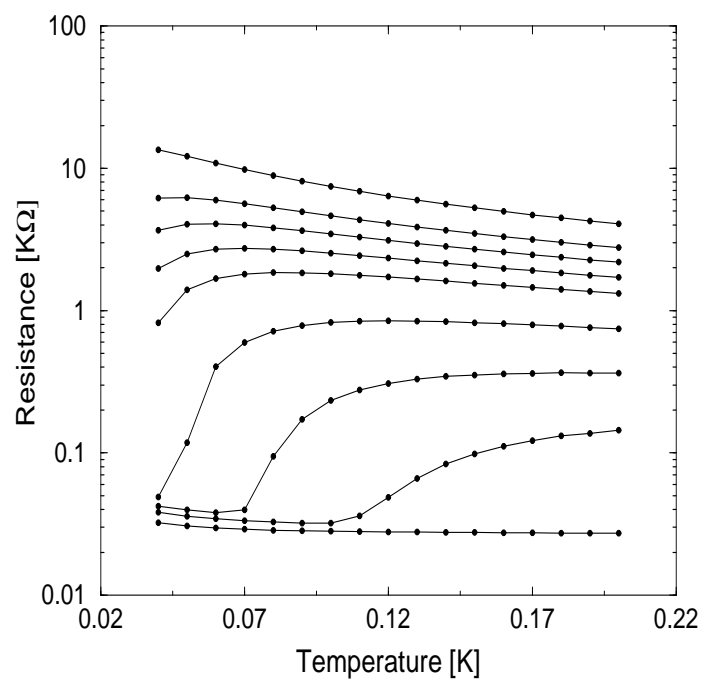

FIG. 1. Temperature dependence of the "zero-bias" resistance at $g=0.4$. From top to bottom, $E_{J} / E_{C}=0.07$, $0.56,0.84,1.12,1.4,1.96,2.52,3.0$ and 4.0 .

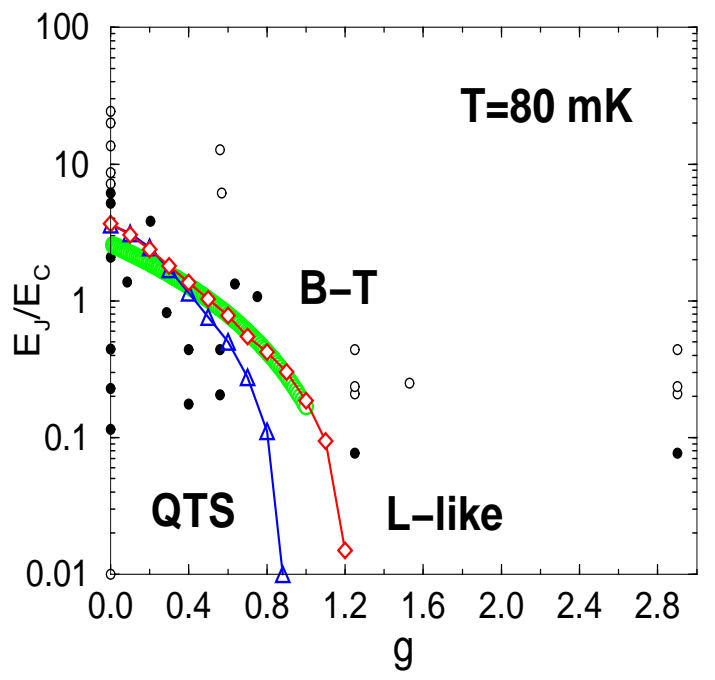

FIG. 2. Phase diagram of shunted Josephson junction at $T=80 \mathrm{mK}$. The phase boundary lies between the insulator-like (solid circles) and superconductor-like (open circles) samples experimentally found in $[7,8]$. The thick line is the band theory(B-T), the triangle the QTS theory and the diamond is due to the present theory $(\mathrm{L}$ like).

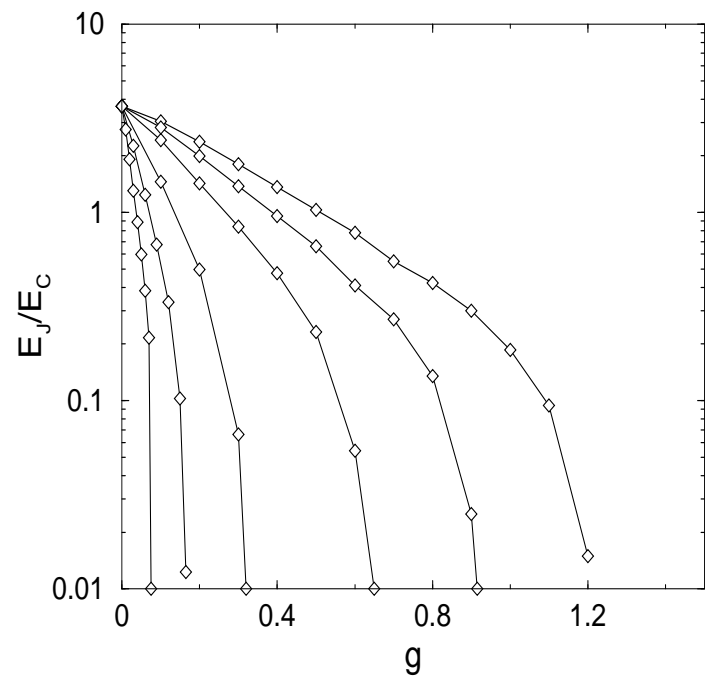

FIG. 3. Temperature dependence of the superconductor-insulator phase boundary in the $E_{J} / E_{C}-g$ plane. From right to left, $T=$ $80,60,40,20,10$, and $5 \mathrm{mK}$. 